\title{
Value of conventional magnetic resonance imaging texture analysis in the differential diagnosis of benign and borderline/ malignant phyllodes tumors of the breast
}

\author{
Xiaoguang Li ${ }^{1}$, Nianping Jiang ${ }^{1}$, Chunlai Zhang ${ }^{1}$, Xiangguo Luo ${ }^{1}$, Peng Zhong ${ }^{2}$ and Jingqin Fang ${ }^{1 *}$
}

\begin{abstract}
Background: The purpose of this study was to determine the potential value of magnetic resonance imaging (MRI) texture analysis (TA) in differentiating between benign and borderline/malignant phyllodes tumors of the breast.

Methods: The preoperative MRI data of 25 patients with benign phyllodes tumors (BPTs) and 19 patients with borderline/malignant phyllodes tumors (BMPTs) were retrospectively analyzed. A gray-level histogram and gray-level cooccurrence matrix (GLCM) were used for TA with fat-suppressed T2-weighted imaging (FS-T2WI), diffusionweighted imaging (DWI), apparent diffusion coefficient (ADC) images, and 2- and 7-min postcontrast T1W images on dynamic contrast-enhanced MRI (DCE-T1WI 2 min and DCE-T1WI $I_{\text {min }}$ ) between BPTs and BMPTs. Independent sample t-test and Mann-Whitney $U$ test were performed for intergroup comparison. A regression model was established by using binary logistic regression analysis, and receiver operating characteristic (ROC) curve analysis was carried out to evaluate diagnostic efficiency.

Results: For ADC images, the texture parameters angular second moment (ASM), correlation, contrast, entropy and the minimum gray values of $A D C$ images ( $A D C_{\text {Minimum }}$ ) showed significant differences between the BPT group and BMPT group (all $p<0.05$ ). The parameter entropy of FS-T2WI and the maximum gray values and kurtosis of the tumor solid region of DCE-T1WI $\mathrm{Imin}_{\text {min }}$ also showed significant differences between these two groups. Except for $\mathrm{ADC}_{\text {Minimum, }}$ angular second moment of FS-T2WI (FS-T2WI $\mathrm{ASM}_{\text {, }}$, and the maximum gray values of DCE-T1WI $\mathrm{Imin}_{\text {min }}$ (DCE-T1WI ${ }_{7 \text { min-Maximum }}$ ) of the tumor solid region, the AUC values of other positive texture parameters mentioned above were greater than 0.75 . Binary logistic regression analysis demonstrated that the contrast of ADC images ( $\left.A D C_{\text {contrast }}\right)$ and entropy of FS-T2WI (FS-T2W| $\left.\left.\right|_{\text {Entropy }}\right)$ could be considered independent texture variables for the differential diagnosis of BPTs and BMPTs. Combined, the AUC of these parameters was 0.891 (95\% Cl: 0.793-0.988), with a sensitivity of $84.2 \%$ and a specificity of up to $89.0 \%$.
\end{abstract}

Conclusion: Texture analysis could be helpful in improving the diagnostic efficacy of conventional MR images in differentiating BPTs and BMPTs.

Keywords: Phyllodes tumors, Magnetic resonance imaging, Texture analysis, Differential diagnosis

\footnotetext{
* Correspondence: jingain0405@163.com

'Department of Radiology, Daping Hospital, Army Medical University, Chongqing 400042, China

Full list of author information is available at the end of the article
}

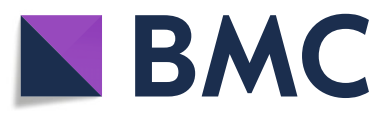

() The Author(s). 2021 Open Access This article is licensed under a Creative Commons Attribution 4.0 International License, which permits use, sharing, adaptation, distribution and reproduction in any medium or format, as long as you give appropriate credit to the original author(s) and the source, provide a link to the Creative Commons licence, and indicate if changes were made. The images or other third party material in this article are included in the article's Creative Commons licence, unless indicated otherwise in a credit line to the material. If material is not included in the article's Creative Commons licence and your intended use is not permitted by statutory regulation or exceeds the permitted use, you will need to obtain permission directly from the copyright holder. To view a copy of this licence, visit http://creativecommons.org/licenses/by/4.0/ The Creative Commons Public Domain Dedication waiver (http://creativecommons.org/publicdomain/zero/1.0/) applies to the data made available in this article, unless otherwise stated in a credit line to the data. 


\section{Introduction}

Phyllodes tumors (PTs) are rare breast fibroepithelial tumors and are classified as benign, borderline and malignant according to the characteristics of the stromal components [1]. Each of these three grades is considered to have a very different biological behavior. Surgery is a fundamental treatment for PTs, and different surgical approaches are commonly selected based on the histologic grade of the tumor [2-4]. In the clinic, BPTs are usually treated with local excision, while extensive excision, including mastectomy, is necessary to reduce recurrence for BMPTs $[5,6]$. Therefore, an accurate preoperative diagnosis and qualitative grading are conducive to the selection of the surgical procedure. Biopsy is the basis of an accurate preoperative diagnosis for breast diseases. However, due to the complex composition and obvious heterogeneity of PTs, it is difficult to obtain representative tissues by percutaneous needle biopsy alone, which could lead to low accuracy in the pathological diagnosis [7].

Magnetic resonance imaging (MRI), with its high sensitivity and relatively high specificity, has become an important imaging method in the diagnosis of breast diseases. However, previous studies have found that the conventional MRI findings of BPTs and BMPTs overlap $[8,9]$, and it is very difficult for physicians to subjectively identify them without obvious morphological features. Current studies on the diagnostic grading ability of functional MRI parameters, such as ADC value, for PT grading are contradictory $[10,11]$, and DCE-MRI findings, such as enhancement pattern or TIC, are not of great value in predicting the histologic grade of PTs $[11,12]$. In addition, MR spectroscopy, which can reflect tumor metabolites, has been unable to conclusively distinguish benign from borderline or malignant PTs to date [8]. Therefore, it is of importance to improve the diagnostic performance of MRI by changing the existing image analysis methods.

Texture analysis (TA) is a method used for the quantitative analysis of image grayscale distribution features and the relationship between pixels and spatial features. Compared with conventional imaging methods, TA can provide objective and additional quantitative image information on lesions independent of the subjective judgment and experience of clinicians or radiologists, adding potential clinical value [13]. Recently, computer-aided TA has been used for the diagnosis and treatment response and prognostic evaluations of cancer patients [14]. However, few studies have used conventional MRI TA to grade PTs. The purpose of this study was to determine the diagnostic performance of conventional MRI TA in differentiating between BPTs and BMPTs.

\section{Methods}

\section{Patients}

We retrospectively reviewed the MRI data of fifty-one patients with surgically proven primary PTs admitted to our hospital between January 2013 and March 2020. 44 patients were enrolled in this study. The exclusion criteria included (1) MRI images with poor quality; (2) implants in one or both breasts; and (3) MRI images acquired after surgery, chemotherapy or radiotherapy. The patient ages ranged from 31 to 75 years (mean $48.55 \pm 10.75$ years). There were 25 cases of BPTs, 16 cases of borderline PTs, and 3 cases of malignant PTs based on the pathological results. The patient flowchart is illustrated in Fig. 1.

\section{Imaging protocol}

All patients were examined with a $1.5 \mathrm{~T}$ MRI scanner (Siemens Magnetom Aera, Germany) in the standard prone position using an 8-channel dedicated breast coil. Axial T1WI (SE, TR $=8.6 \mathrm{~ms}, \mathrm{TE}=4.7 \mathrm{~ms}$ ) and fatsuppressed T2WI (FSE, TR $=5600 \mathrm{~ms}, \mathrm{TE}=57 \mathrm{~ms}$ ) were obtained. DWI was performed with a spin echo-echo planar imaging (SE-EPI) sequence with two b values $(0$ and $\left.1000 \mathrm{~s} / \mathrm{mm}^{2}\right)$ in 3 orthogonal directions. The imaging parameters were as follows: $\mathrm{TR}=3300 \mathrm{~ms}, \mathrm{TE}=94 \mathrm{~ms}$, flip angle $=90^{\circ}$, layer thickness $=5 \mathrm{~mm}$, matrix $=128 \times 128$, and FOV $=320 \mathrm{~mm} \times 320 \mathrm{~mm}$. Following DWI, DCE-MRI was performed with a 3D fat-suppressed $\mathrm{T} 1$ fast-field echo sequence $(\mathrm{TR}=4.62 \mathrm{~ms}, \mathrm{TE}=1.75 \mathrm{~ms}$, layer thickness $=1.5$ $\mathrm{mm}$, interlayer spacing $=0$, FOV $=360 \mathrm{~mm} \times 360 \mathrm{~mm}$, and matrix $=384 \times 320$ ) before and five times after the injection of $0.1 \mathrm{mmol} / \mathrm{kg}$ gadopentetate dimeglumine (Omniscan, GE Healthcare, Ireland). Subsequently, 7-phase DCE-T1W images were acquired.

\section{Imaging analysis}

All FS-T2WI, DWI, ADC, DCE-T1WI $\mathrm{I}_{2 \min }$ and DCE$\mathrm{T}_{1} \mathrm{WI}_{7 \text { min }}$ tumor images were exported in DICOM format from the PACS system and then imported to RadiAnt software (https://www.radiantviewer.com/) to render them in BMP format with identical window widths and window levels. ImageJ software (https://rsb. info.nih.gov/ij/) was used for image TA (Fig. 1), and all the data were analyzed separately by two radiologists (CL.Z. and XG.L., with 10 and 15 years of experience in breast imaging, respectively). The regions of interest (ROIs) were extracted as follows: ROIs were placed on the image containing the maximal tumor area for all MR images and included necrotic, cystic, and hemorrhagic areas. The tumor solid region was delineated on DCE$\mathrm{T} 1 \mathrm{WI}_{7 \text { min }}$ (Fig. 2). Finally, the gray-level histogram and gray-level cooccurrence matrix (GLCM) parameters of all the ROIs were automatically measured by the software [15]. Definitions and formulas for the histogram 
Fifty-one patients underwent breast MRI and were confirmed as phyllodes tumors by pathology;

January 2013 to March 2020

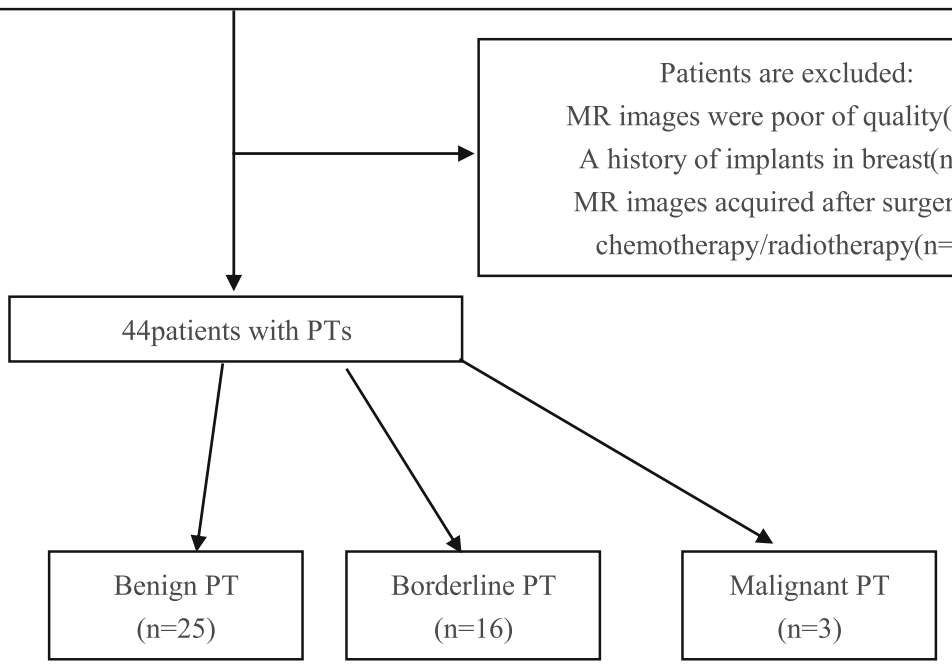

Fig. 1 Flowchart for the case accrual process

and GLCM parameters are shown in Table 1. GLCM is a spatial domain statistical technique that calculates second- and higher-order statistics for the number of paired $(i, j)$ occurrences for which a gray level $i$ is spaced away from a gray level $j$ by a distance (d) and along a direction
( $\theta)$ [16]. In this study, the relationship between the pixels of the GLCMs was set with $d=1$ and $\theta=0^{\circ}$ $[17,18]$. The final histogram and GLCM parameter values of each lesion were the mean of the measured results from the two radiologists.
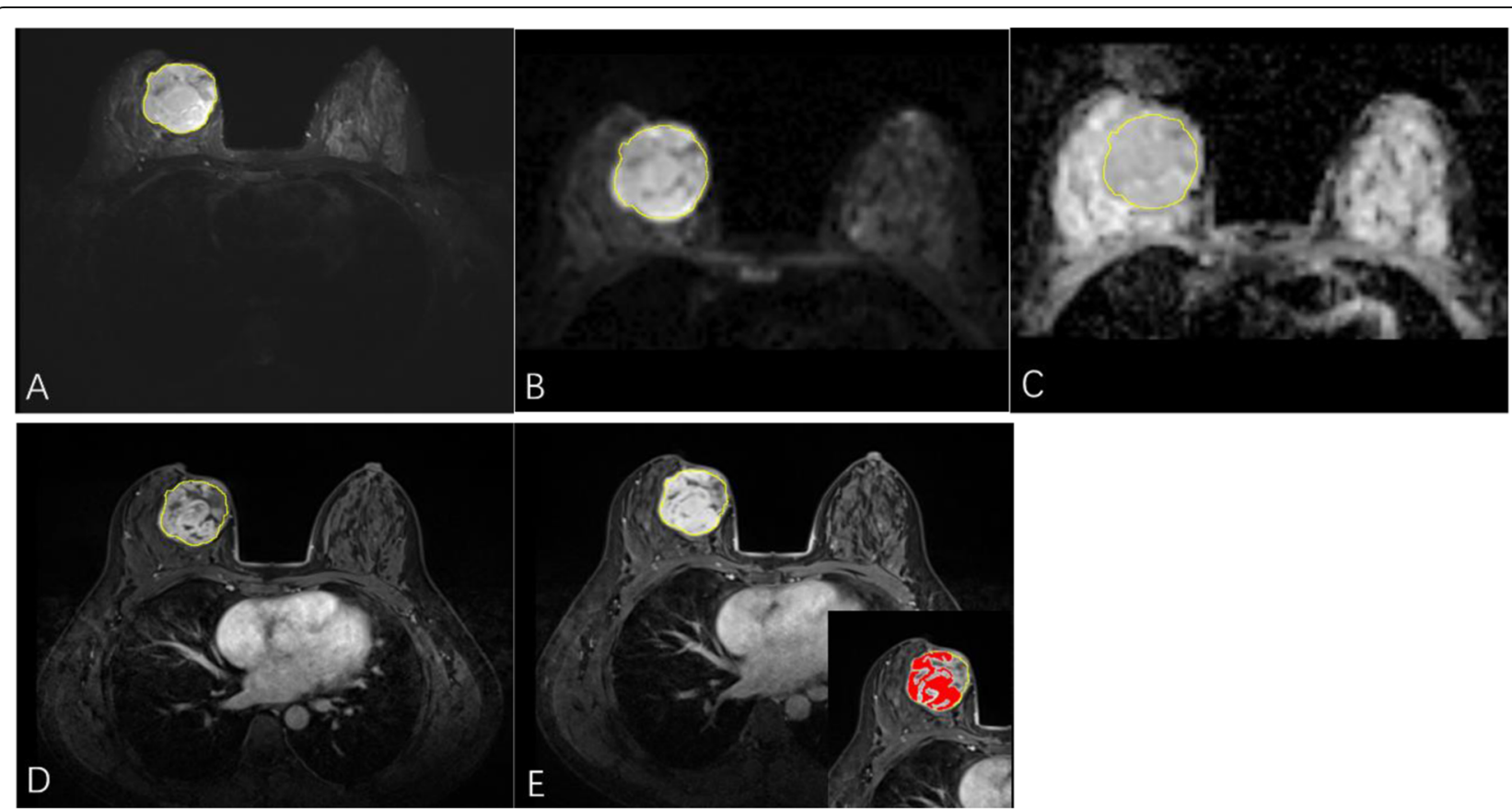

Fig. 2 Schematic diagram of the ROI. ImageJ software was used to select the layer with the maximum tumor are from the FS-T2WI (a) and manually delineate the tumor boundary as much as possible, which was automatically copied to the DWI (b), ADC image (c), DCE-T1WI 2 min (d), and DCE$\mathrm{T}_{1 \mathrm{~W}} \mathrm{I}_{7 \text { min }}(\mathbf{e})$ of the same tumor layer. Note that the red part in the lower right corner of the DCE-T1Wl $\left.\right|_{7 \text { min }}$ (e) represents the solid tumor region 
Table 1 Representative gray-level histogram and gray-level co-occurrence matrix texture features

\begin{tabular}{|c|c|c|}
\hline Texture parameters & Qualitative description & Mathematical description \\
\hline \multicolumn{3}{|l|}{ Histogram parameters } \\
\hline Mean & Mean gray-level value & Mean $=\sum k \sum^{k^{*} g(k)}$ \\
\hline Minimum & Minimum gray-level value & $\operatorname{Min}=\operatorname{Min}(k)$ \\
\hline Maximum & Maximum gray-level value & $\operatorname{Max}=\operatorname{Max}(k)$ \\
\hline Skewness & Measure of histogram symmetry & Skew $=\sigma^{-4} \Sigma_{k}(k-\mu)^{4} * g(k)-3$ \\
\hline Kurtosis & Measure of histogram flatness & Kurt $=\sigma^{-3} \Sigma_{k}(k-\mu)^{4} * g(k)$ \\
\hline \multicolumn{3}{|l|}{ GLCM parameters } \\
\hline Angular Second Moment (ASM) & Certainty of gray-level co-occurrence & $\operatorname{ASM}=\sum_{i, j} f(i, j)^{2}$ \\
\hline Contrast & Intensity contrast between pixel and its neighbor & $\mathrm{CON}=\sum_{i, j}|i-j|^{2} f(i, j)$ \\
\hline Correlation & Linear gray-level dependence & $\operatorname{COR}=\sum_{i, j} \frac{\left(i-\mu_{i}\right)^{*}\left(j-\mu_{j}\right)^{*} f(i, j)}{\left(1+(i-j)^{2}\right)}$ \\
\hline Inverse difference moment (IDM) & $\begin{array}{l}\text { Local homogeneity in gray-level } \\
\text { co-occurrence }\end{array}$ & $\mathrm{IDM}=\sum_{i, j} \frac{f(i, j)}{\left(1+(i-j)^{2}\right)}$ \\
\hline Entropy & $\begin{array}{l}\text { Uncertainty of gray-level } \\
\text { co-occurrence }\end{array}$ & ENT $=-\sum_{i, j} f(i, j)^{*} \log (f(i, j))$ \\
\hline
\end{tabular}

\section{Statistical analysis}

Statistical analyses were performed using IBM SPSS version 21.0 (IBM Corporation, New York). KolmogorovSmirnov and Levene tests were used to determine the normality and homogeneity of variance, respectively, of all measurement data. The independent sample $t$-test and the Mann-Whitney $U$ test were used for data with normal and nonnormal distributions, respectively. Bonferroni's correction was used to adjust $p$ values for multiple parameter comparisons [19]. For texture parameters with significant differences, the group was taken as the dependent variable, logistic regression analysis was performed for multiparameter joint analysis, and the predicted value of the computational model was used to draw the receiver operator characteristic (ROC) curve. The efficacy (sensitivity, specificity, 95\% confidence interval and $p$ value) of each individual texture parameter and of the combined parameters in the identification of the two groups was determined with the maximum parameter value of the Youden index [(sensitivity + specificity) -1 ] as the threshold. $p<0.05$ indicated a statistically significant difference.

\section{Results}

\section{Comparisons of texture parameters of different images} between the BPT and BMPT groups

As illustrated in Tables 2 and 3, for FS-T2WI, the GLCM texture parameters ASM and entropy were significantly different between the two groups (both $p<$ $0.05)$. However, no histogram parameters showed significant intergroup differences. For ADC images, the GLCM parameters ASM, correlation, contrast, entropy and histogram parameter $\mathrm{ADC}_{\text {Minimum }}$ showed significant differences (all $\mathrm{p}<0.05$ ). For DWI and DCE-T1WI ${ }_{2 \min }$, none of the histogram or GLCM parameters showed significant differences (all $\mathrm{p}>0.05$ ). For DCE-T1WI $\mathrm{I}_{7 \mathrm{~min}}$, none of the histogram or GLCM parameters of tumor overall region showed significant differences. The maximum gray values and kurtosis of the tumor solid region showed significant differences (all $\mathrm{p}<0.05$ ); however, no GLCM parameters showed significant differences for this region of the tumor (all $\mathrm{p}>0.05$ ).

\section{Diagnostic efficacy of MRI texture analysis in differentiating between BPTs and BMPTs}

The parameters with significant differences between the two groups were further analyzed by ROC curve analysis. Those parameters with an $\mathrm{AUC}>0.75$ were $\mathrm{ADC}_{\mathrm{ASM}}$, $\mathrm{ADC}_{\text {Contrast, }}, \mathrm{ADC}_{\text {Correlation}}, \mathrm{ADC}_{\text {Entropy }}, \mathrm{FS}-\mathrm{T} 2 \mathrm{WI} \mathrm{I}_{\text {Entropy }}$, and kurtosis of DCE-T1WI $\mathrm{I}_{7 \text { min }}\left(\mathrm{DCE}-\mathrm{T} 1 \mathrm{WI} \mathrm{I}_{7 \text { min-Kurtosis }}\right)$ of the tumor solid region. Among them, $\mathrm{ADC}_{\text {Contrast }}$ had the highest differential diagnostic efficiency, with an AUC of 0.815 , a sensitivity of $84.2 \%$ and a specificity of $76.0 \%$. Binary logistic regression analysis revealed that both $\mathrm{ADC}_{\mathrm{Con}-}$ trast and FS-T2WI $I_{\text {Entropy }}$ showed significant differences between the two groups $(p<0.05)$ and were thus regarded as independent variables. Then, the following regression eq. (RE) was obtained: $P=-13.616+0.067 A D C_{\text {Contrast }}+$ $1.341 F S-T 2 W I_{\text {Entropy }}$. The ROC curve of the combined texture parameters from the logistic regression model was plotted, and its identification efficiency was shown to be better than that of each individual texture parameter. The AUC was 0.891 (95\% CI: 0.793-0.988), with a sensitivity of $84.2 \%$ and a specificity of up to $89.0 \%$ (Table 4 , Fig. 3).

\section{Discussion}

TA is a radiomics technique that can help reveal the potential heterogeneity within tumor lesions and provide quantitative and objective information on conventional MR images in the clinic [20]. First-order TA is performed 
Table 2 Comparisons of histogram parameters of ADC images, FS-T2WI, DWI, DCE-T1WI 2 min and DCE-T1WI 1 min between the two groups, respectively

\begin{tabular}{|c|c|c|c|c|}
\hline Parameters & BPTs $(n=25)$ & BMPTs $(n=19)$ & $P$ value & $\begin{array}{l}\text { Adjusted } \\
P \text { value }\end{array}$ \\
\hline$A D C_{\text {Mean }}$ & $174.790 \pm 26.849$ & $164.122 \pm 31.669$ & 0.234 & 0.229 \\
\hline$A D C_{\text {Minimum }}$ & $138.154 \pm 37.446$ & $108.842 \pm 38.312$ & 0.022 & 0.018 \\
\hline$A D C_{\text {Maximum }}$ & $216.240 \pm 33.286$ & $218.263 \pm 29.790$ & 0.836 & 0.833 \\
\hline$A D C_{\text {Skewness }}$ & $0.097 \pm 0.804$ & $0.147 \pm 0.858$ & 0.844 & 0.842 \\
\hline$A D C_{\text {kurtosis }}$ & $0.797 \pm 1.553$ & $0.988 \pm 1.598$ & 0.693 & 0.688 \\
\hline${ }^{\mathrm{a}}$ FS-T2WI Mean & $131.680(95.702,160.844)$ & $147.528(124.366,154.154)$ & 0.387 & 0.568 \\
\hline FS-T2WI $I_{\text {Minimum }}$ & $50.600 \pm 35.344$ & $54.263 \pm 32.527$ & 0.726 & 0.722 \\
\hline${ }^{a}$ FS-T2WI Maximum & $234.000(175.500,255.000)$ & $244.000(222.000,255.000)$ & 0.259 & 0.264 \\
\hline FS-T2WI $\left.\right|_{\text {skewness }}$ & $0.054 \pm 0.571$ & $0.228 \pm 0.516$ & 0.304 & 0.298 \\
\hline${ }^{a}$ FS-T2W| Kurtosis & $0.372(0.097,1.701)$ & $0.110(-0.318,0.918)$ & 0.110 & 0.121 \\
\hline DWI $I_{\text {Mean }}$ & $166.476 \pm 30.833$ & $160.400 \pm 36.953$ & 0.556 & 0.549 \\
\hline$\left.\mathrm{DW}\right|_{\text {Minimum }}$ & $93.640 \pm 34.946$ & $100.053 \pm 33.766$ & 0.544 & 0.538 \\
\hline${ }^{a}$ DWI $\left.\right|_{\text {Maximum }}$ & $245.000(229.500,247.500)$ & $245.000(229.000,252.000)$ & 0.601 & 0.893 \\
\hline${ }^{a}$ DWI $\left.\right|_{\text {Skewness }}$ & $-0.254(-0.441,0.385)$ & $0.192(-0.151, .443)$ & 0.132 & 0.405 \\
\hline${ }^{a}$ DWI Kurtosis & $-0.152(-0.511,0.503)$ & $-0.102(-0.523,0.463)$ & 0.972 & 0.704 \\
\hline$\left(D C E-T 1 W I_{2 \min }\right)_{\text {Mean }}$ & $132.006 \pm 35.1049$ & $125.593 \pm 36.500$ & 0.558 & 0.552 \\
\hline a $\left(\mathrm{DCE}-\mathrm{T} 1 \mathrm{~W} \mathrm{I}_{2 \mathrm{~min}}\right)_{\text {Minimum }}$ & $58.000(45.000,91.500)$ & $36.000(25.000,50.000)$ & 0.048 & 0.128 \\
\hline$\left(\mathrm{DCE}-\mathrm{T} 1 \mathrm{~W} \mathrm{I}_{2 \mathrm{~min}}\right)_{\text {Maximum }}$ & $200.320 \pm 32.983$ & $202.526 \pm 39.141$ & 0.840 & 0.838 \\
\hline$\left(\mathrm{DCE}-\left.\mathrm{T} 1 \mathrm{~W}\right|_{2 \mathrm{~min}}\right)_{\text {Skewness }}$ & $0.0409 \pm 0.56225$ & $-0.0359 \pm 0.79452$ & 0.709 & 0.704 \\
\hline a $\left(\mathrm{DCE}-\left.\mathrm{T} 1 \mathrm{~W}\right|_{2 \text { min }}\right)_{\text {Kurtosis }}$ & $-0.041(-0.380,0.813)$ & $-0.018(-0.706,0.789)$ & 0.731 & 0.493 \\
\hline$\left(\mathrm{DCE}-\mathrm{T} 1 \mathrm{~W} \mathrm{I}_{7 \min }\right)_{\text {Mean }}$ & $179.321 \pm 35.092$ & $166.457 \pm 41.996$ & 0.275 & 0.270 \\
\hline$\left(\mathrm{DCE}-\mathrm{T} 1 \mathrm{WI} \mathrm{I}_{7 \mathrm{~min}}\right)_{\text {Minimum }}$ & $91.080 \pm 56.3116$ & $76.632 \pm 52.2007$ & 0.389 & 0.383 \\
\hline${ }^{a}\left(\mathrm{DCE}-\mathrm{T} 1 \mathrm{~W} \mathrm{I}_{7 \mathrm{~min}}\right)_{\text {Maximum }}$ & $243.000(232.000,250.500)$ & $232.000(214.000,241.000)$ & 0.037 & 0.145 \\
\hline$\left(\mathrm{DCE}-\left.\mathrm{T} 1 \mathrm{~W}\right|_{7 \mathrm{~min}}\right)_{\text {skewness }}$ & $-0.647 \pm 0.708$ & $-0.793 \pm 0.811$ & 0.527 & 0.521 \\
\hline${ }^{\mathrm{a}}\left(\mathrm{DCE}-\mathrm{T} 1 \mathrm{~W} \mathrm{I}_{7 \text { min }}\right)_{\text {Kurtosis }}$ & $0.691(-0.229,1.932)$ & $1.210(0.285,2.972)$ & 0.420 & 0.414 \\
\hline $\begin{array}{l}\text { a }\left(\mathrm{DCE}-\mathrm{T} 1 \mathrm{~W} \mathrm{I}_{7 \min }\right)_{\text {Mean }} \\
\text { of tumor solid region }\end{array}$ & $200.935(173.433,211.289)$ & $184.417(150.868,206.929)$ & 0.260 & 0.224 \\
\hline$\left(\mathrm{DCE}-\mathrm{T} 1 \mathrm{~W} \mathrm{I}_{7 \mathrm{~min}}\right)_{\text {Minimum }}$ of tumor solid region & $142.080 \pm 45.118$ & $123.000 \pm 41.919$ & 0.160 & 0.157 \\
\hline a $\left(\mathrm{DCE}-\left.\mathrm{T} 1 \mathrm{~W}\right|_{7 \min }\right)_{\text {Maximum }}$ of tumor solid region & $240.000(231.500,247.000)$ & $209.000(231.000,238.000)$ & 0.024 & 0.028 \\
\hline$\left(\mathrm{DCE}-\left.\mathrm{T} 1 \mathrm{~W}\right|_{7 \min }\right)_{\text {skewness }}$ of tumor solid region & $-0.023 \pm 0.6125$ & $-0.282 \pm 0.919$ & 0.268 & 0.263 \\
\hline a $\left(\mathrm{DCE}-\mathrm{T} 1 \mathrm{~W} \mathrm{I}_{7 \text { min }}\right)_{\text {Kurtosis }}$ of tumor solid region & $0.161(-0.189,0.620)$ & $1.178(0.507,2.677)$ & 0.004 & 0.009 \\
\hline
\end{tabular}

Note: Plus-minus values are means \pm SD. ${ }^{a}$ The data were expressed as median (quantile range, QR), and intergroup comparison was analyzed with Mann-Whitney $\mathrm{U}$ text. ADC: apparent diffusion coefficient; FS-T2WI fat-suppressed T2-weighted imaging; DWI diffusion weighted imaging; $D C E-T 1 W I_{2 m i n}$ and DCE-T1WI ${ }_{7 \text { min }} 2$ - and 7-min postcontrast T1W images on DCE-MRI; ASM angular second moment; IDM inverse difference moment

through the gray-level histogram, which mainly describes the distribution of individual gray intensity values. Generally, the ROI is decomposed into single values representing gray-signal intensity: the mean value, maximum value, minimum value, skewness, and kurtosis. A higher gray value indicates a brighter ROI area. The ADC image histogram is the most popular method for analyzing MRI tumor histograms, and a series of parameters obtained from the ADC image histogram for retrospective analysis have good repeatability [21]. In our study, both the $\mathrm{ADC}_{\text {Mean }}$ and $\mathrm{ADC}_{\text {Maximum }}$ gray values of $\mathrm{BPTs}$ were larger than those of BMPTs; however, there was no significant difference between these two groups, similar to the conclusion made in the study by Guo et al. [12]. In his study, there was no significant difference in the ADC values between the BPT and BMPT groups for the mean ROI-w (the whole-tumor ROI) values or for the 10th, 25th, 50th and 75th percentile values from the ROI-w histogram.

Previous studies have found that the minimum ADC value has the best accuracy in differentiating between malignant and benign breast masses [22, 23]. In this 
Table 3 Comparisons of GLCM parameters of ADC images, FS-T2WI, DWI, DCE-T1WI $\left.\right|_{2 m i n}$ and DCE-T1WI 7 min between the two groups, respectively

\begin{tabular}{|c|c|c|c|c|}
\hline Parameter & BPTs $(n=25)$ & BMPTs $(n=19)$ & $P$ value & Adjusted $P$ value \\
\hline$\overline{\operatorname{ADC}_{\operatorname{ASM}}\left(10^{-4}\right)}$ & $32.402 \pm 20.370$ & $17.242 \pm 10.652$ & 0.003 & 0.007 \\
\hline$A D C_{\text {Contrast }}$ & $39.516 \pm 15.473$ & $71.30 \pm 31.43$ & 0.000 & 0.000 \\
\hline${ }^{\mathrm{a}} \mathrm{ADC}$ Correlation & $23.866(13.465,40.467)$ & $9.577(5.320,12.695)$ & 0.002 & 0.004 \\
\hline $\operatorname{ADC}_{\text {IDM }}\left(10^{-4}\right)$ & $0.273 \pm 0.070$ & $0.236 \pm 0.045$ & 0.056 & 0.057 \\
\hline ADC Entropy & $6.356 \pm 0.750$ & $6.990 \pm 0.542$ & 0.003 & 0.005 \\
\hline${ }^{\mathrm{a}} \mathrm{FS}-\mathrm{T} 2 \mathrm{WI} \mathrm{ISSM}_{\mathrm{AS}}\left(10^{-4}\right)$ & $10.218(7.374,13.788)$ & $6.054(4.924,8.588)$ & 0.017 & 0.023 \\
\hline FS-T2WI Contrast & $284.043 \pm 163.702$ & $262.016 \pm 89.184$ & 0.600 & 0.594 \\
\hline${ }^{a}$ FS-T2WI Correlation & $6.390(3.824,10.029)$ & $4.255(3.864,5.710)$ & 0.166 & 0.337 \\
\hline a FS-T2W| $\left.\right|_{\text {IDM }}\left(10^{-4}\right)$ & $0.128(0.096,0.157)$ & $0.126(0.115,0.153)$ & 0.325 & 0.195 \\
\hline${ }^{\mathrm{a}}$ FS-T2W| & $7.071(6.760,7.602)$ & $7.745(7.364,7.990)$ & 0.004 & 0.011 \\
\hline$\left.{ }^{\mathrm{a}} \operatorname{DWI}\right|_{\mathrm{ASM}}\left(10^{-4}\right)$ & $10.573(7.104,17.995)$ & $9.214(7.660,14.154)$ & 0.822 & 0.913 \\
\hline DWI $\left.\right|_{\text {Contrast }}$ & $127.468 \pm 70.053$ & $108.686 \pm 78.431$ & 0.407 & 0.401 \\
\hline${ }^{a} \mathrm{DWI}$ Correlation & $4.785(3.970,6.325)$ & $5.340(4.159,8.704)$ & 0.374 & 0.781 \\
\hline$\left.D W\right|_{\mid D M}\left(10^{-4}\right)$ & $0.179 \pm 0.080$ & $0.193 \pm 0.083$ & 0.560 & 0.553 \\
\hline DWI $\left.\right|_{\text {Entropy }}$ & $7.070 \pm 0.627$ & $7.176 \pm 0.573$ & 0.566 & 0.560 \\
\hline${ }^{\mathrm{a}}\left(\mathrm{DCE}-\mathrm{T} 1 \mathrm{~W} \mathrm{I}_{2 \mathrm{~min}}\right)_{\mathrm{ASM}}\left(10^{-4}\right)$ & $6.671(4.723,8.487)$ & $4.454(3.295,9.788)$ & 0.387 & 0.734 \\
\hline${ }^{\mathrm{a}}\left(\mathrm{DCE}-\mathrm{T} 1 \mathrm{~W} \mathrm{I}_{2 \mathrm{~min}}\right)_{\text {Correlation }}$ & $147.562(109.739,278.188)$ & $142.800(112.616,358.782)$ & 0.785 & 0.477 \\
\hline a $\left(\mathrm{DCE}-\mathrm{T} 1 \mathrm{WI} \mathrm{I}_{2 \mathrm{~min}}\right)_{\text {Contrast }}$ & $5.624(3.773,10.021)$ & $5.839(4.304,9.361)$ & 0.610 & 0.666 \\
\hline a $\left(\mathrm{DCE}-\left.\mathrm{T} 1 \mathrm{~W}\right|_{2 \min }\right)_{\mathrm{IDM}}\left(10^{-4}\right)$ & $0.1319(0.1108,0.1689)$ & $0.137(0.096,0.185)$ & 0.731 & 0.816 \\
\hline$\left(\mathrm{DCE}-\left.\mathrm{T} 1 \mathrm{~W}\right|_{2 \mathrm{~min}}\right)_{\text {Entropy }}$ & $7.529 \pm 0.678$ & $7.741 \pm 0.635$ & 0.296 & 0.291 \\
\hline a $(\text { DCE-T1W| } 7 \text { min })_{\text {ASM }}\left(10^{-4}\right)$ & $7.9483(5.086,14.714)$ & $9.201(5.506,12.687)$ & 0.661 & 0.730 \\
\hline a $\left(\mathrm{DCE}-\mathrm{T} 1 \mathrm{~W} \mathrm{I}_{7 \mathrm{~min}}\right)_{\text {Correlation }}$ & $188.777(151.258,350.693)$ & $205.771(43.797,296.260)$ & 0.991 & 0.711 \\
\hline${ }^{a}\left(\mathrm{DCE}-\left.\mathrm{T} 1 \mathrm{~W}\right|_{7 \min }\right)_{\text {Contrast }}$ & $3.668(2.910,5.350)$ & $4.623(3.475,5.598)$ & 0.222 & 0.191 \\
\hline$\left(\mathrm{DCE}-\left.\mathrm{T} 1 \mathrm{~W}\right|_{7 \min }\right)_{\mathrm{IDM}}\left(10^{-4}\right)$ & $0.169 \pm .0708$ & $0.171 \pm 0.059$ & 0.907 & 0.906 \\
\hline$\left(\mathrm{DCE}-\left.\mathrm{T} 1 \mathrm{~W}\right|_{7 \min }\right)_{\text {Entropy }}$ & $7.360 \pm 0.772$ & $7.562 \pm 0.610$ & 0.354 & 0.348 \\
\hline $\begin{array}{l}\text { a }\left(\text { DCE-T1WI }\left.\right|_{7 \min }\right) \text { ASM } \\
\text { of tumor solid region }\left(10^{-4}\right)\end{array}$ & $9.306(5.4594,15.915)$ & $10.669(5.257,13.941)$ & 0.400 & 0.561 \\
\hline $\begin{array}{l}\text { a }\left(\mathrm{DCE}-\mathrm{T} 1 \mathrm{~W} \mathrm{I}_{7 \min }\right)_{\text {Correlation }} \\
\text { of tumor solid region }\end{array}$ & $189.607(129.702,350.693)$ & $209.754(141.136,296.260)$ & 0.934 & 0.735 \\
\hline $\begin{array}{l}\text { a }\left(\mathrm{DCE}-\left.\mathrm{T} 1 \mathrm{~W}\right|_{7 \min }\right)_{\text {Contrast }} \\
\text { of tumor solid region }\end{array}$ & $3.772(3.082,5.563)$ & $4.712(3.331,9.056)$ & 0.314 & 0.222 \\
\hline $\begin{array}{l}\left(\mathrm{DCE}-\left.\mathrm{T} 1 \mathrm{~W}\right|_{7 \min }\right)_{I \mathrm{DM}} \\
\text { of tumor solid region }\left(10^{-4}\right)\end{array}$ & $0.175 \pm 0.074$ & $0.169 \pm 0.057$ & 0.770 & 0.766 \\
\hline $\begin{array}{l}\left.\text { (DCE-T1WI } I_{7 \min }\right)_{\text {Entropy }} \\
\text { of tumor solid region }\end{array}$ & $7.230 \pm 0.760$ & $7.495 \pm 0.654$ & 0.232 & 0.227 \\
\hline
\end{tabular}

Note: Plus-minus values are means \pm SD. ${ }^{\text {a }}$ The data were expressed as median (quantile range, QR), and intergroup comparison was analyzed with Mann-Whitney $\mathrm{U}$ text. $A D C$ apparent diffusion coefficient; $F S$-T2WI fat-suppressed T2-weighted imaging; $D W I$ diffusion weighted imaging; $D C E-T 1 W I_{2 \min }$ and $D C E-T 1 W I_{7 m i n} 2-$ and 7-min postcontrast T1W images on DCE-MRI; ASM angular second moment; IDM inverse difference moment

study, we found that the $\mathrm{ADC}_{\text {Minimum }}$ gray value of BPTs was significantly higher than that of BMPTs, which indicates that the $\mathrm{ADC}_{\text {Minimum }}$ gray value can better display areas with higher cellular density than the $\mathrm{ADC}_{\text {Mean }}$ gray value. The mean $\mathrm{ADC}$ value based on conventional hot spot ROIs or the histogram ROI only represents the average level of the data, which might be limited to PTs with considerable heterogeneity. However, it should be emphasized that the $\mathrm{ADC}_{\text {Minimum }}$ gray value may be more susceptible to outliers from noise, artifacts, adjacent structures and the partial volume effect; therefore, great care should be taken when delineating ROIs [24]. Kurtosis and skewness are statistics reflecting the distribution of the image gray values. The steeper the kurtosis, the steeper the distribution is compared with the normal distribution; the greater the absolute value of skewness, the greater the skewness of the distribution is $[25,26]$. In our study, neither of these two parameters 
Table 4 Receiver operating characteristic curve analysis for the positive texture variables between the BPTs and BMPTs

\begin{tabular}{lllll}
\hline Parameter & AUC & $\mathbf{9 5 \% C l}$ & Sensitivity & Specificity \\
\hline ADC $_{\text {Minimum }}$ & 0.705 & $0.504-0.829$ & $70.4 \%$ & $65.0 \%$ \\
ADC $_{\text {ASM }}$ & 0.756 & $0.609-0.903$ & $73.7 \%$ & $72.0 \%$ \\
ADC Contrast & 0.815 & $0.680-0.949$ & $84.2 \%$ & $76.0 \%$ \\
ADC Correlation & 0.781 & $0.642-0.920$ & $84.2 \%$ & $72.0 \%$ \\
ADC Entropy $_{\text {FS-T2W| }}$ & 0.785 & $0.647-0.923$ & $78.9 \%$ & $76.0 \%$ \\
FS-T2W| $_{\text {Entroy }}$ & 0.712 & $0.546-0.877$ & $73.7 \%$ & $76.0 \%$ \\
$\begin{array}{l}\text { DCE-T1W| } \\
\text { of solid tumor region }\end{array}$ & 0.756 & $0.607-0.905$ & $73.7 \%$ & $78.0 \%$ \\
$\begin{array}{l}\text { DCE-T1W| } \\
\text { of solid tumin-Kurtosis }\end{array}$ & 0.758 & $0.600-0.916$ & $78.9 \%$ & $76.0 \%$ \\
Combined parameters & 0.891 & $0.793-0.988$ & $84.2 \%$ & $89.0 \%$ \\
\hline
\end{tabular}

Note: $A D C$ apparent diffusion coefficient; FS-T2WI fat-suppressed T2-weighted imaging; $A S M$ angular second moment; IDM inverse difference moment. $D C E$ $\mathrm{T}_{1 \mathrm{WI}} \mathrm{I}_{\min }$ 7-min postcontrast T1W images on DCE-MRI

was able to distinguish BPTs from BMPTs, indicating that they are of little importance in distinguishing the two groups based on the morphological changes in ADC gray histograms.

The GLCM is one of the important methods used in second-order TA. The GLCM can describe the spatial relationship between voxels by analyzing the gray distribution of pixels and the surrounding spatial domain [27]. The texture parameter ASM reflects homogeneity, the value of which is quite high when the image has perfect homogeneity or when the pixel intensity is very similar. Correlation reflects the linear dependency of the gray levels of neighboring pixels, and high values can be obtained for regions of similar gray levels [28]. In this study, the GLCM derived from ADC images showed that the ASM and correlation values of BPTs were significantly higher than those of BMPTs. This indicates that BPTs have a more uniform gray distribution and stronger texture regularity than BMPTs on ADC images. Contrast reflects the amount of gray-level variation in an image, where a high contrast value indicates the presence of noise or a wrinkled texture in an image. The increased contrast values of BMPTs suggest more noise or wrinkled textures in malignant PT lesions, which may be associated with the local heterogeneous intensity. Entropy represents the amount of information needed for image compression. A higher entropy value represents a greater loss of image information and a more complex image texture [29]. In this study, BMPTs had a higher entropy value than BPTs, suggesting that BMPTs lose more image information and thereby have increased complexity and heterogeneity.

FS-T2WI is one of the more important sequences for MRI TA, which may be related to the relatively long

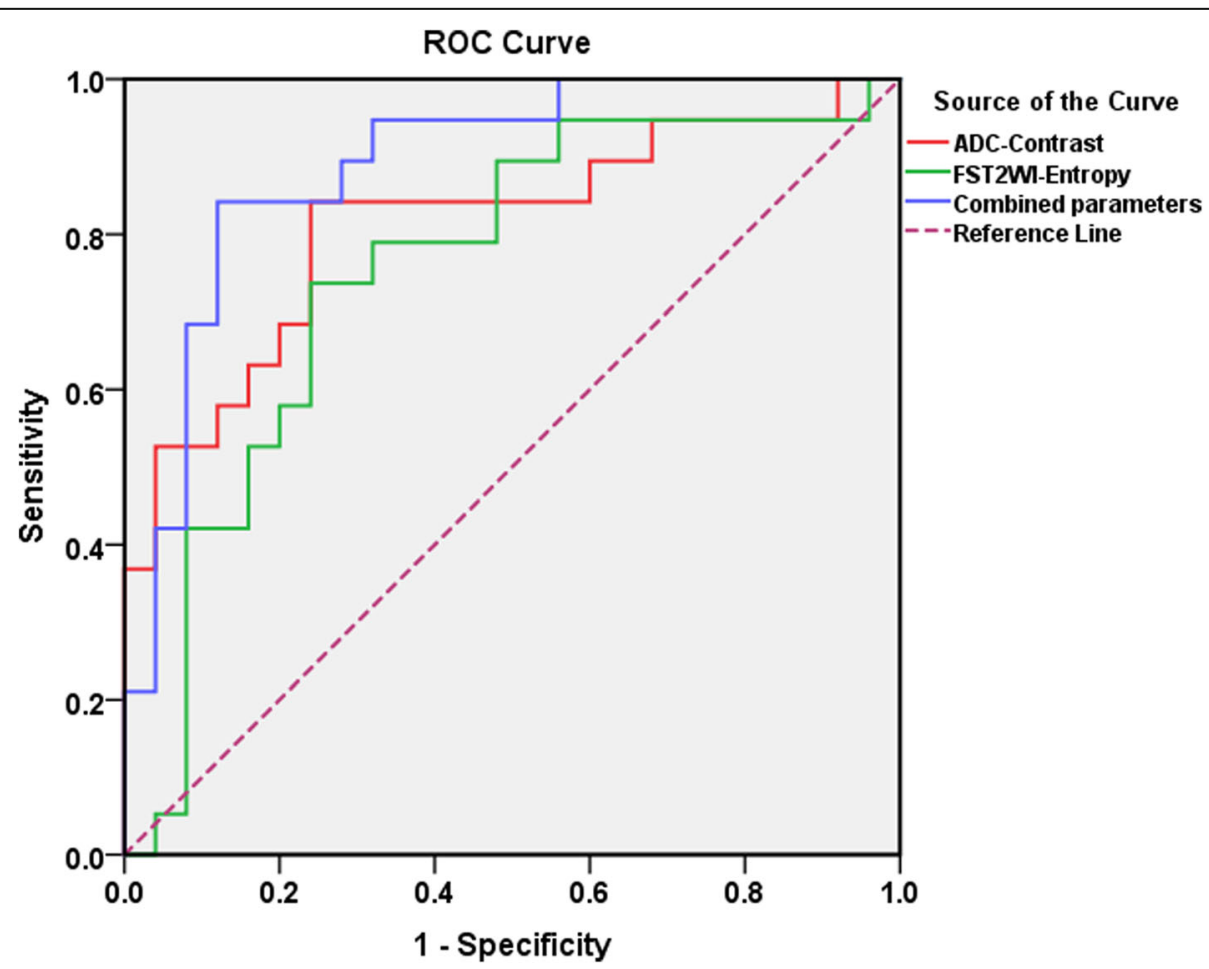

Fig. 3 ROC curves of independent variables and the combination of texture parameters from the logistic regression model for differentiating BPTs from BMPTs 
time of echo (TE) of the sequence, increasing the interorganizational contrast and making the image contain more texture features of diagnostic significance [30]. In this study, we did not find any FS-T2WI gray histogram parameter that could distinguish BPTs from BMPTs. However, we did find significant differences in the GLCM parameters ASM and entropy between the two groups. The entropy of BPTs was significantly lower than that of BMPTs, indicating that the FS-T2WI texture of BMPTs is more complex and heterogeneous than that of BPTs. This may be related to the fact that BMPTs are more prone to allogenic metaplasia, which further complicates their internal composition. To some extent, these heterogeneous structures can also explain why the ASM of BMPTs was significantly lower than that of BPTs. It is worth mentioning that in empirical imaging analysis, we tend to consider that the degree of diffusion limitation of malignant PTs on DWI is more obvious and that the signal is higher than that of benign PTs. A previous study showed that the accuracy of DWI in characterizing lesions by using $b$ values $=0 \mathrm{~s} / \mathrm{mm}^{2}$ and $1000 \mathrm{~s} / \mathrm{mm}^{2}$ was the best when breast lesions were identified on 1.5-T MRI [31]. Therefore we attempted to verify whether high $b$ value $\left(b=1000 \mathrm{~s} / \mathrm{mm}^{2}\right)$ DWI TA could be of importance in differentiating between BPTs and BMPTs. However, the results were disappointing, showing that none of the histogram and GLCM parameters could differentiate between BPTs and BMPTs. We conjecture that this might be related to the nature of high $b$ value DWI, in which the signal-to-noise ratio (SNR) can be reduced, along with image information.

Of the 7 phases of DCE-MRI scanning performed, we selected DCE-T1WI ${ }_{2 \min }$ and DCE-T1WI ${ }_{7 \text { min }}$ for study, mainly because the contrast agent had just entered the tumor at 2 min of DCE-T1WI, and the texture comparisons were substantial; furthermore, at $7 \mathrm{~min}$ of DCET1WI, all components of the tumor could demonstrate significant contrast with the surrounding glandular tissues [32]. The results showed that the histograms of the parameters mean, minimum and maximum gray value of DCE-T1WI ${ }_{2 \min }$ and DCE-T1WI ${ }_{7 \min }$ (both solid and overall region) were higher in the BPT group than in the BMPT group, and only the maximum gray value of DCE-T1WI ${ }_{7 \min }$ for tumor solid region showed significant differences between the two groups after Bonferroni's correction. This indicates a higher enhancement degree for the tumor solid region in BPTs than in BMPTs. Additionally, the kurtosis of the tumor solid region in the BPT group was significantly lower than that in the BMPT group, which suggests a more uniform signal from the tumor solid region in BPTs on DCET1WI ${ }_{7 m i n}$. Previous studies [33] have shown that the GLCM based on DCE-MRI can better reflect tumor heterogeneity, and texture differences may reflect the potential pathological subtypes of breast cancer. However, we found no significant difference in GLCM parameters between the BPT and BMPT groups, either in the tumor overall region on $\mathrm{DCE}-\mathrm{T} 1 \mathrm{WI}_{2 \min }$ and DCE-T1WI $\mathrm{I}_{7 \min }$ or in the tumor solid region on DCE$\mathrm{T} 1 \mathrm{WI}_{7 \text { min }}$.

In this study, although multiple texture parameters were statistically significant in differentiating between BPTs and BMPTs, by drawing the ROC curves, we found that the GLCM parameters derived from ADC images had better diagnostic performance, in which the AUC of contrast reached more than 0.8, with the highest sensitivity (84.2\%) and specificity (76.0\%). Furthermore, binary logistic regression analysis showed that the texture parameters $\mathrm{ADC}_{\text {Contrast }}$ and FS-T2WI $\mathrm{Wntropy}_{\text {were }}$ independent variables in differentiating BPTs from BMPTs. ROC curve analysis showed that the combination of these two texture parameters had excellent diagnostic efficiency, with an AUC of 0.891 , an optimal sensitivity of 0.842 and a specificity of 0.890 , all of which were better than the diagnostic efficiency of individual sequences and single parameters.

There are several limitations to our study that deserve discussion. First, it is inevitable that the size of the sample would be small, especially for malignant PTs. Second, this study was a single-center retrospective study with no external data validation, and in particular, the differences in MRI scanning protocols between different studies may lead to deviations in the image data. Third, only DWI based on $b=1000 / \mathrm{mm}^{2}$ were used for texture analysis, and other DWI with different $\mathrm{b}$ values should be examined in the future. Fourth, our study was performed using 1.5 T MRI systems for acquiring DWI, and the possibility of differing results using a higher magnetic field ( $3 \mathrm{~T})$ cannot be excluded. Fifth, we analyzed only the two-dimensional features of the maximum surface of the tumor but did not obtain the threedimensional features of the whole tumor. Sixth, only first-order histograms and second-order GLCM parameters were used for the differentiational diagnosis of PTs; whether higher-order texture parameters, such as the run-length matrix (RLM) and absolute gradient matrix (ARM), are helpful in the identification of BPTs and BMPTs is worth further discussion.

\section{Conclusion}

This study conducted a texture analysis based on FST2WI, DWI $\left(b=1000 / \mathrm{mm}^{2}\right), \quad$ ADC images, DCE$\mathrm{T}_{1 \mathrm{WI}} \mathrm{I}_{2 \min }$ and DCE-T1WI $\mathrm{Wmin}_{\text {min }}$ to explore their diagnostic value in the preoperative classification of PTs. The results showed that the texture parameters that could aid in the differentiation between BPTs and BMPTs were mainly derived from the GLCM analysis of ADC images, among which $\mathrm{ADC}_{\text {Contrast }}$ had the highest differential efficacy. In 
addition, we found that combined multiparameter TA from multiple images could greatly improve the efficiency of the identification of BPTs and BMPTs. Thus, MRI texture analysis may be used as an image-processing tool that is worthy of further evaluation in the differential diagnosis of BPTs and BMPTs.

\begin{abstract}
Abbreviations
MRI: Magnetic resonance imaging; DCE-MRI: Dynamic contrast-enhanced MRI; FS-T2WI: T2-weighted imaging with fat suppression; DWI: Diffusionweighted imaging; ADC: Apparent diffusion coefficient; TA: Texture analysis; BPTs: Benign phyllodes tumors; BMPTs: Borderline/malignant phyllodes tumors; ROC: Receiver operating characteristic; ROI: Region of interest; AUC: The area under the curve; GLCM: Gray-level cooccurrence matrix; ASM: Angular second moment; IDM: Inverse difference moment
\end{abstract}

\section{Acknowledgments}

Not applicable.

\section{Authors' contributions}

FJQ conducted study design. JNP contributed to data collection, ZCL and LUO-XG contributed to data analysis, ZP provided the histopathological results, LI-XG prepared the original draft and FJQ reviewed and edited the final manuscript. The author(s) read and approved the final manuscript.

\section{Funding}

This work was supported by the Fund of Chongqing Clinical Research Centre of Imaging and Nuclear Medicine (CSTC2015YFPT-gcjsyjzx0175) and Central government guide the development of local science and technology special fund (YDZX20175000004270).

\section{Availability of data and materials}

The datasets supporting the conclusions of this article are included within the article.

\section{Declarations}

\section{Ethics approval and consent to participate}

This study was approved by the institutional ethics committee of Da-ping Hospital of Army Medical University (Ratification NO: 2019(159)), and informed consent was waived due to the retrospective character of the study.

\section{Consent for publication}

Not applicable.

\section{Competing interests}

The authors declare that they have no competing interest.

\section{Author details}

'Department of Radiology, Daping Hospital, Army Medical University, Chongqing 400042, China. ${ }^{2}$ Department of Pathology, Daping Hospital, Army Medical University, Chongqing 400042, China.

Received: 14 November 2019 Accepted: 4 March 2021

Published online: 12 March 2021

\section{References}

1. Bendifallah S, Canlorbe G. [common benign breast tumors including fibroadenoma, phyllodes tumors, and papillary lesions: guidelines]. J Gynecol Obstet. Biol Reprod (Paris). 2015;44(10):1017-29.

2. Spitaleri G, Toesca A, Botteri E, Bottiglieri L, Rotmensz N, Boselli S, et al. Breast phyllodes tumor: a review of literature and a single center retrospective series analysis. Crit Rev Oncol Hematol. 2013;88(2):427-36.

3. Tan PH, Thike AA, Tan WJ, Thu MM, Busmanis I, Li H, et al. Predicting clinical behaviour of breast phyllodes tumours: a nomogram based on histological criteria and surgical margins. J Clin Pathol. 2012;65(1):69-76.

4. Tan BY, Acs G, Apple SK, Badve S, Bleiweiss IJ, Brogi E, et al. Phyllodes tumours of the breast: a consensus review. Histopathology. 2016;68(1):5-21.
5. Pezner RD, Schultheiss TE, Paz IB. Malignant phyllodes tumor of the breast: local control rates with surgery alone. Int J Radiat Oncol Biol Phys. 2008; 71(3):710-3.

6. Li J, Tsang JY, Chen C, Chan SK, Cheung SY, Wu C, et al. Predicting outcome in mammary Phyllodes tumors: relevance of Clinicopathological features. Ann Surg Oncol. 2019;26(9):2747-58.

7. Zhang Y, Kleer CG. Phyllodes tumor of the breast: Histopathologic features, differential diagnosis, and molecular/genetic updates. Arch Pathol Lab Med. 2016;140(7):665-71.

8. Plaza MJ, Swintelski C, Yaziji H, Torres-Salichs M, Esserman LE. Phyllodes tumor: review of key imaging characteristics. Breast Dis. 2015;35(2):79-86.

9. Tan $H$, Zhang $S$, Liu H, Peng W, Li R, Gu Y, et al. Imaging findings in phyllodes tumors of the breast. Eur J Radiol. 2012;81(1):e62-9.

10. Kawashima H, Miyati T, Ohno N, Ohno M, Inokuchi M, Ikeda H, et al. Differentiation between phyllodes tumours and fibroadenomas using intravoxel incoherent motion magnetic resonance imaging: comparison with conventional diffusion-weighted imaging. Br J Radiol. 2018;91(1084): 20170687

11. Yabuuchi H, Soeda H, Matsuo Y, Okafuji T, Eguchi T, Sakai S, et al. Phyllodes tumor of the breast: correlation between MR findings and histologic grade. Radiology. 2006;241(3):702-9.

12. Guo Y, Tang W, Kong Q, Liang Y, Han X, Zheng B, et al. Can whole-tumor apparent diffusion coefficient histogram analysis be helpful to evaluate breast phyllode tumor grades? Eur J Radiol. 2019;114(3):25-31.

13. Chitalia RD, Kontos D. Role of texture analysis in breast MRI as a cancer biomarker: a review. J Magn Reson Imaging. 2019;49(4):927-38.

14. Davnall F, Yip CSP, Ljungqvist G, Selmi M, Ng F, Sanghera B, et al. Assessment of tumor heterogeneity: an emerging imaging tool for clinical practice? Insights Into Imaging. 2012;3(6):573-89.

15. Xu X, Hu H, Su G, Liu H, Hong X, Shi H, et al. Utility of histogram analysis of ADC maps for differentiating orbital tumors. Diagnostic Intervention Radiol (Ankara, Turkey). 2016;22(2):161-7.

16. Kono K, Hayata R, Murakami S, Yamamoto M, Kuroki M, Nanato K, et al. Quantitative distinction of the morphological characteristic of erythrocyte precursor cells with texture analysis using gray level co-occurrence matrix. J Clin Lab Anal. 2018;32(1):e22175.

17. Wang B, Liu G, Fan W, Zhang X, Lu Y, Chen Z. Value of texture feature analysis in the differential diagnosis of hepatic cyst and Hemangioma in magnetic resonance imaging. Zhongguo Yi Xue Ke Xue Yuan Xue Bao. 2017:39(2):169-76.

18. Parekh V, Jacobs MA. Radiomics: a new application from established techniques. Expert Rev Precision Med Drug Dev. 2016;1 (2):207-26.

19. Kim HS, Kim J, Yoon YC, Choe BK. Tumor spatial heterogeneity in myxoidcontaining soft tissue using texture analysis of diffusion-weighted MRI. PLoS One. 2017;12(7):e0181339.

20. Gillies RJ, Kinahan PE, Hricak H. Radiomics: images are more than pictures. They Are Data Radiology. 2016;278(2):563-77.

21. Song YS, Park CM, Lee SM, Park SJ, Cho HR, Choi SH, et al. Reproducibility of histogram and texture parameters derived from intravoxel incoherent motion diffusion-weighted MRI of FN13762 rat breast carcinomas. Anticancer Res. 2014:34(5):2135-44.

22. Hirano M, Satake H, Ishigaki S, Ikeda M, Kawai H, Naganawa S. Diffusionweighted imaging of breast masses: comparison of diagnostic performance using various apparent diffusion coefficient parameters. AJR Am J Roentgenol. 2012:198(3):717-22.

23. Suo S, Zhang K, Cao M, Suo X, Hua J, Geng X, et al. Characterization of breast masses as benign or malignant at 3.0T MRI with whole-lesion histogram analysis of the apparent diffusion coefficient. J Magn Reson Imaging. 2016;43(4):894-902.

24. Kang Y, Choi SH, Kim YJ, Kim KG, Sohn CH, Kim JH, et al. Gliomas: histogram analysis of apparent diffusion coefficient maps with standard- or high-bvalue diffusion-weighted MR imaging--correlation with tumor grade. Radiology. 2011;261(3):882-90.

25. Downey K, Riches SF, Morgan VA, Giles SL, Attygalle AD, Ind TE, et al. Relationship between imaging biomarkers of stage I cervical cancer and poor-prognosis histologic features: quantitative histogram analysis of diffusion-weighted MR images. AJR Am J Roentgenol. 2013;200(2): $314-20$

26. Lin Y, Li H, Chen Z, Ni P, Zhong Q, Huang H, et al. Correlation of histogram analysis of apparent diffusion coefficient with uterine cervical pathologic finding. AJR Am J Roentgenol. 2015;204(5):1125-31. 
27. Wibmer A, Hricak H, Gondo T, Matsumoto K, Veeraraghavan $H$, Fehr $D$, et al. Haralick texture analysis of prostate MRI: utility for differentiating noncancerous prostate from prostate cancer and differentiating prostate cancers with different Gleason scores. Eur Radiol. 2015;25(10):2840-50.

28. Chen X, Wei X, Zhang Z, Yang R, Zhu Y, Jiang X. Differentiation of trueprogression from pseudoprogression in glioblastoma treated with radiation therapy and concomitant temozolomide by GLCM texture analysis of conventional MRI. Clin Imaging. 2015;39(5):775-80.

29. Henderson S, Purdie C, Michie C, Evans A, Lerski R, Johnston M, et al. Interim heterogeneity changes measured using entropy texture features on T2-weighted MRI at 3.0 T are associated with pathological response to neoadjuvant chemotherapy in primary breast cancer. Eur Radiol. 2017; 27(11):4602-11.

30. Li Z, Mao Y, Huang W, Li H, Zhu J, Li W, et al. Texture-based classification of different single liver lesion based on SPAIR T2W MRI images. BMC Med Imaging. 2017;17(1):42.

31. Dorrius MD, Dijkstra H, Oudkerk M, Sijens PE. Effect of $b$ value and preadmission of contrast on diagnostic accuracy of 1.5-T breast DWl: a systematic review and meta-analysis. Eur Radiol. 2014;24(11):2835-47.

32. Pinker $\mathrm{K}$, Helbich TH, Morris EA. The potential of multiparametric MRI of the breast. Br J Radiol. 2017;90(1069):20160715.

33. Fan M, Li H, Wang S, Zheng B, Zhang J, Li L. Radiomic analysis reveals DCEMRI features for prediction of molecular subtypes of breast cancer. PLoS One. 2017;12(2):e0171683.

\section{Publisher's Note}

Springer Nature remains neutral with regard to jurisdictional claims in published maps and institutional affiliations.

Ready to submit your research? Choose BMC and benefit from:

- fast, convenient online submission

- thorough peer review by experienced researchers in your field

- rapid publication on acceptance

- support for research data, including large and complex data types

- gold Open Access which fosters wider collaboration and increased citations

- maximum visibility for your research: over $100 \mathrm{M}$ website views per year

At $\mathrm{BMC}$, research is always in progress.

Learn more biomedcentral.com/submissions 